\title{
Identification and clinical impact of potentially actionable somatic oncogenic mutations in solid tumor samples
}

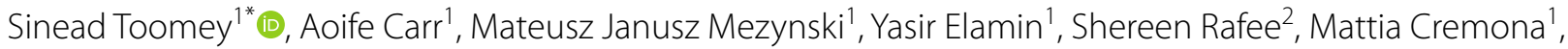
Clare Morgan', Stephen Madden³, Khairun I. Abdul-Jalil', Kathy Gately², Angela Farrelly', Elaine W. Kay ${ }^{4}$, Susan Kennedy ${ }^{5,6}$, Kenneth O'Byrne ${ }^{2,7}$, Liam Grogan ${ }^{8}$, Oscar Breathnach ${ }^{8}$, Patrick G. Morris ${ }^{8}$, Alexander J. Eustace ${ }^{1}$, Joanna Fay ${ }^{4}$, Robert Cummins ${ }^{4}$, Anthony O'Grady ${ }^{4}$, Roshni Kalachand ${ }^{1}$, Norma O'Donovan ${ }^{9}$, Fergal Kelleher ${ }^{10}$, Aine O'Reilly ${ }^{8}$, Mark Doherty ${ }^{8}$, John Crown ${ }^{9,10}$ and Bryan T. Hennessy ${ }^{1,8}$

\begin{abstract}
Background: An increasing number of anti-cancer therapeutic agents target specific mutant proteins that are expressed by many different tumor types. Successful use of these therapies is dependent on the presence or absence of somatic mutations within the patient's tumor that can confer clinical efficacy or drug resistance.

Methods: The aim of our study was to determine the type, frequency, overlap and functional proteomic effects of potentially targetable recurrent somatic hotspot mutations in 47 cancer-related genes in multiple disease sites that could be potential therapeutic targets using currently available agents or agents in clinical development.

Results: Using MassArray technology, of the 1300 patient tumors analysed 571 (43.9\%) had at least one somatic mutation. Mutations were identified in 30 different genes. KRAS (16.5\%), PIK3CA (13.6\%) and BRAF (3.8\%) were the most frequently mutated genes. Prostate (10.8\%) had the lowest number of somatic mutations identified, while no mutations were identified in sarcoma. Ocular melanoma (90.6\%), endometrial (72.4\%) and colorectal (66.4\%) tumors had the highest number of mutations. We noted high concordance between mutations in different parts of the tumor (94\%) and matched primary and metastatic samples (90\%). KRAS and BRAF mutations were mutually exclusive. Mutation co-occurrence involved mainly PIK3CA and PTPN11, and PTPN11 and APC. Reverse Phase Protein Array (RPPA) analysis demonstrated that PI3K and MAPK signalling pathways were more altered in tumors with mutations compared to wild type tumors.
\end{abstract}

Conclusions: Hotspot mutational profiling is a sensitive, high-throughput approach for identifying mutations of clinical relevance to molecular based therapeutics for treatment of cancer, and could potentially be of use in identifying novel opportunities for genotype-driven clinical trials.

\footnotetext{
*Correspondence: sineadtoomey@rcsi.ie

${ }^{1}$ Medical Oncology Lab, Department of Molecular Medicine, Royal

College of Surgeons in Ireland, RCSI Smurfit Building, Beaumont Hospital, Dublin, Ireland

Full list of author information is available at the end of the article
}

\begin{abstract}
Background
Traditionally most cancers were categorised in terms of their tissue of origin, the size and nodal status of the primary tumor, and the presence of metastatic lesions. For solid tumors, the origin of the tumor was generally the deciding factor in assessing treatment options, and patients were usually assigned to different treatment options based on primary tumor diagnosis or site,
\end{abstract}


histological subtype, nodal status, hormone receptor and HER2 status for breast and gastro-oesophageal cancers, and KRAS and EGFR status for colorectal and lung cancers, respectively.

With the discovery that many tumors contain mutations within oncogenes or tumor suppressor genes that may predict responses to targeted anti-cancer therapies, genomic profiling to support treatment decisions is used in some settings. Well established examples include KIT mutations which are present in $\sim 85 \%$ of gastro-intestinal stromal tumors [1], EGFR mutations that have been identified in $\sim 15 \%$ of non-small cell lung cancers [2], and lung and colorectal cancers with mutations in the KRAS oncogene [3]. Several agents have been developed to target these molecules including the tyrosine kinase inhibitor imatinib, which induces clinical responses in gastrointestinal stromal tumors that harbour KIT mutations [4], and erlotinib and gefitinib which are effective in non-small cell lung cancers with mutations or insertions/ deletions in EGFR [2, 5]. BRAF V600E mutations are found in approximately half of all cutaneous melanomas, and the use of BRAF inhibitors in these patients has been shown to improve survival [6-8]. In metastatic colorectal cancers the use of EGFR inhibitors in combination with conventional chemotherapy significantly improved survival $[9,10]$. Furthermore, afatinib and osimertinib have recently been approved for the treatment of EGFR mutated non-small cell lung cancers [11, 12], while the combination of BRAF and MEK inhibitors improve overall survival in melanoma [13]. However, the presence of mutations in proteins other than the intended therapeutic target can affect the response to a particular therapy. For example, lung and colorectal cancers with mutations in $K R A S$ or $B R A F$ do not respond to treatment with antiEGFR therapies [3].

Oncogene mutations do not usually occur randomly, but are more frequent in certain genomic regions [14]. Because genomic aberrations can predict responsiveness to targeted therapies, profiling cancer mutations will allow a greater understanding of the pathways involved in driving the cancers growth, and ultimately allow for the genetic and/or molecular characteristics of the tumor to play a role in determining the choice of therapy. This process will maximise the efficacy of treatment while minimising undesirable side effects resulting from altered drug metabolism due to the patient's genetic background. Genomically guided therapies may be of particular use in treating rare tumors, where very large randomised trials are often impractical [15]. Currently most genomic technologies to profile samples for the clinical selection of patients for targeted therapies assess the mutational status of one or a few genes (e.g. pyrosequencing) or investigate a specific histologic phenotype [immunohistochemistry and fluorescence in situ hybridisation (FISH)]. These approaches can miss multiple alterations that are also potentially targetable, and other alterations that may be markers of resistance to standard therapies. While next generation sequencing (NGS) has made it possible to test multiple genes simultaneously, tumor molecular profiling by NGS remains challenging in the clinical setting. Whole genome or exome sequencing is not feasible for many clinical labs due to the large amount of data required to detect low level variants and the time and bioinformatics expertise needed to analyse the data.

In the present study, we have used MassArray technology, a high-throughput mass spectrometry-based technique which enables sensitive and rapid somatic mutation profiling in solid tumor samples. The MassArray technology makes it possible to analyse multiple hotspot mutations within 3 days, and negates the need for complex bioinformatic analysis. Initial studies using this technology showed it to be highly advantageous for somatic mutation profiling. A study by Thomas et al. used a somatic mutation panel, comprising of 238 oncogene mutations in 17 oncogenes, to screen 1000 human tumor samples from 17 types of solid tumors. Relevant mutations were confirmed in $30 \%$ of the samples, and novel mutations were detected that had not been previously reported due to the sensitivity of this method [16]. Since then numerous other studies have demonstrated the feasibility of using MassArray to identify actionable mutations for the purpose of implementing genomedriven oncology programs [17-20], and the technology has recently been approved as a clinical diagnostic platform [18].

Emerging evidence suggests that many of the genomic aberrations currently used to guide the selection of targeted therapies within specific disease contexts may also occur in other cancer types. The identification and targeting of these biomarkers provides an opportunity to extend the benefits of personalised medicine to a larger population of patients. The aim of this study was to determine the type, frequency, overlap and functional proteomic effects of potentially targetable recurrent somatic hotspot mutations in 47 cancer-related genes in multiple disease sites that could be potential therapeutic targets using currently available agents or agents in clinical development.

\section{Methods}

\section{Tumor samples}

Formalin-fixed paraffin embedded (FFPE) tumor samples were obtained from tumor banks under the auspices of Institutional Review Board-approved protocols at Beaumont Hospital Dublin, Ireland (613 samples), St. 
Vincent's University Hospital, incorporating the Royal Victoria Eye and Ear Hospital, Dublin, Ireland (470 samples), and St. James's Hospital, Dublin, Ireland (217 samples). Tumor selection was based on the most prevalent cancers treated in our hospitals and the availability of sufficient tissue from the tumor. In total, the tumors of 1300 patients were evaluated. More than one region of the tumor was evaluated from 50 patients, and a matched metastatic sample was evaluated from 30 patients. All patients were diagnosed between January 1994 and October 2014.

\section{Sample preparation}

In each case, up to $6 \times 10 \mu \mathrm{M}$ sections were cut from the paraffin block. Sandwich hematoxylin and eosin staining was performed on the first and last sections and checked by an experienced pathologist to ensure that tumor was present throughout the sections. If the tumor content was lower than $50 \%$, tumor area was macrodissected. DNA was extracted using an All Prep DNA FFPE kit (Qiagen) as per manufacturer's instructions. DNA concentration was calculated using the Qubit ds DNA Kit. A total of $10 \mathrm{ng}$ DNA from each sample was subjected to beta-globin gene (300 base pairs) polymerase chain reaction and analysed on a $1.5 \%$ Agarose gel to verify DNA quality. Only DNA samples with successful $\beta$-globin amplification were subjected to further analysis.

\section{Mutation analysis}

Mass-spectrometry-based single nucleotide polymorphism genotyping technology (Agena Biosciences, Hamburg, Germany) was used for identification of hotspot, potentially clinically relevant nonsynonymous somatic mutations (Additional file 1: Table S1). Because these mutations were known to be somatic from previous studies, no germline samples were included in this analysis. The genes were further subdivided by pathway, and include MAPK, PI3K and related pathway genes (Additional file 1: Table S2). Assays were designed using strict assay design parameters optimized for sensitive mutation detection. The panel consisted of 31 multiplex assays capable of detecting 504 somatic hotspot mutations in 47 genes. Ten nanograms (10 ng) of DNA was added to each PCR reaction and DNA was amplified using custom designed PCR primer pools. Unincorporated nucleotides were inactivated using shrimp alkaline phosphatase, and a single base extension reaction was performed using extension primers that hybridise immediately adjacent to the mutations of interest. Salts were removed by adding a cation-exchange resin, before the multiplexed reactions were spotted onto SpectroCHIP II arrays. Matrix chips were analysed on an Agena MassArray MALDI-TOF system.

\section{Protein extraction and RPPA analysis of tumors}

From each tumor, up to $5 \times 10 \mu \mathrm{M}$ sections were cut from the paraffin block. Protein extraction was carried out as previously described [21] using a 20-mM Tris buffer $\mathrm{pH} 9$ containing 2\% SDS and protease inhibitors (Roche Applied Science Cat. \# 04693116001 and 04906845001) lysis buffer.

RPPA analysis was carried out as previously described by us $[22,23]$. The antibodies used are in supplementary data (Additional file 2: Table S3). The data was normalised by protein loading using the entire antibody panel.

\section{Statistical analysis}

Mutation calls for each sample were determined using visual inspection and Typer Software based on mass spectra. Reactions where $>15 \%$ of the resultant mass ran in the mutant sites were scored as positive. Overall survival (OS) was measured in months from the date of diagnosis to the date of death from any cause, and progression free survival (PFS) from the date of diagnosis to the date of documented radiological recurrence or death from any cause in the absence of documented recurrence. The Kaplan-Meier method and the log rank test were used to analyse the associations between survival [progression free survival (PFS) and overall survival (OS)] for groups of interest. The statistical significance of co-occurring mutations was determined using Fisher's exact test. Differences of $\mathrm{p}<0.05$ were considered statistically significant. Statistical analysis was performed with GraphPad Prism version 5.01.

\section{Results}

\section{Sample set and clinicopathologic characteristics}

Archival paraffin embedded tumor tissue from 1300 cancer patients was analysed. Patient characteristics are detailed in Table 1 and Additional file 1: Figure S1. Five hundred and ninety three patients $(45.6 \%)$ were male and 707 (54.4\%) were female. The median age was 63 years (range 19-90). Thirty nine patients had metastatic disease at the time of diagnosis. The tumor types were colorectal $(\mathrm{n}=354,27.2 \%)$, lung $(\mathrm{n}=223,17.2 \%)$, breast $(\mathrm{n}=172$, $13.2 \%)$, prostate $(n=83,6.4 \%)$, melanoma $(n=65,5 \%)$, lymphoma $(n=49,3.8 \%)$, gastric $(n=45,3.5 \%)$, head and neck $(n=45,3.5 \%)$, bladder $(n=38,2.9 \%)$, ocular melanoma $(n=32,2.5 \%)$, endometrial $(n=29,2.2 \%)$, kidney $(\mathrm{n}=28,2.2 \%)$, ovary $(\mathrm{n}=24,1.8 \%)$, brain $(\mathrm{n}=24,1.8 \%)$, oesophagus $(n=22,1.7 \%)$, pancreas $(n=19,1.5 \%)$, liver $(n=16,1.2 \%)$, testis $(n=16,1.2 \%)$, thyroid $(n=13,1 \%)$ and sarcoma $(n=3,0.2 \%)$. Two samples were tested in 80 patients. Of these, 50 patients had two regions of the primary tumor tested, while 30 patients had a primary and 
Table 1 Classification of the samples studied by age and clinical characteristics $(n=1300)$

\begin{tabular}{lc}
\hline Clinical characteristic & Number of patients (\%) \\
\hline Gender & \\
Male & $593(45.6)$ \\
Female & $707(54.4 \%)$ \\
Age at diagnosis & \\
Median (range) & $63(19-90)$ \\
Tumor type & \\
Colorectal & $354(27.2 \%)$ \\
Lung & $223(17.2 \%)$ \\
Breast & $172(13.2 \%)$ \\
Prostate & $83(6.4 \%)$ \\
Melanoma & $65(5 \%)$ \\
Lymphoma & $49(3.8 \%)$ \\
Gastric & $45(3.5 \%)$ \\
Head and neck & $45(3.5 \%)$ \\
Bladder & $38(2.9 \%)$ \\
Ocular melanoma & $32(2.5 \%)$ \\
Endometrial & $29(2.2 \%)$ \\
Kidney & $28(2.2 \%)$ \\
Ovary & $24(1.8 \%)$ \\
Brain & $24(1.8 \%)$ \\
Oesophagus & $22(1.7 \%)$ \\
Pancreas & $19(1.5 \%)$ \\
Liver & $16(1.2 \%)$ \\
Testis & $16(1.2 \%)$ \\
Thyroid & $13(1 \%)$ \\
Sarcoma & $3(0.2 \%)$ \\
Origin of the tumors & \\
Primary tumor & $1166(89.7 \%)$ \\
Metastasis & $44(3.4 \%)$ \\
Unknown & $90(6.9 \%)$ \\
\hline
\end{tabular}

a metastatic site analysed. Patients who had more than one sample tested were considered mutated for a specific gene if mutations were seen in any of the samples tested.

\section{Mutation detection}

Genotyping was performed for 504 somatic hotspot mutations in 47 genes. Among the 1300 patient tumors tested, 571 patients (43.9\%) had at least one potentially targetable alteration. A gene was considered targetable if there is either an approved or investigational therapy targeting the product of the gene either directly or indirectly, although this may not reflect current availability or therapeutic efficacy in different tumor types. Proportionately ocular melanoma had the greatest number of potentially targetable mutations $(90.6 \%, 29 / 32)$, followed by endometrial $(72.4 \%, 21 / 29)$ and colorectal $(66.4 \%, 235 / 354)$ cancers, while liver $(12.5 \%, 2 / 16)$, prostate cancer $(10.8 \%, 9 / 83)$ and sarcoma $(0 \% 0 / 3)$ had the lowest number of potentially targetable mutations (Fig. 1).

The most frequently mutated genes were $K R A S(16.5 \%)$ and PIK3CA (13.6\%). Somatic alterations were also detected in BRAF (3.8\%), PTPN11 (3.3\%), STK11 (2.7\%), NRAS (2.7\%), FBXW7 (2\%), CTNNB1 (1.8\%), APC (1.7\%), GNA11 (1.3\%), PTEN (1\%), HRAS (1\%), CDKN2A (1\%), GNAQ (0.9\%), KIT (0.9\%), MYC (0.6\%), GNAS (0.5\%), ERBB2 (0.4\%), AKT (0.4\%), MAP3K13 (0.3\%), FGFR1 (0.2\%), FGFR2 (0.2\%), TBX3 (0.2\%), NCOR1 $(0.2 \%)$, MAP2K1 (0.2\%), IDH1 (0.2\%), RB1 (0.1\%), CDK4 (0.1\%), MAP2K2 (0.1\%) and FGFR3 (0.1\%) (Fig. 2a). Mutations in KRAS were most frequently identified in colorectal cancers, followed by lung cancers. Mutations in PIK3CA were most frequently identified in breast cancers and lung cancers, while $B R A F$ mutations were most frequently identified in colorectal cancers and melanomas. Full details of the percentage of mutations found in each tumor type are shown in Fig. 2a and Additional file 3: Table S5. When the somatic mutations were subdivided by pathway, $27.9 \%$ of tumors had a MAPK pathway mutation, while $17.6 \%$ of tumors had a PI3K pathway mutation (Fig. 2b). Details of the genes included in each pathway are shown in Additional file 1: Table S2. Analysis using the Cancer Genome Interpreter demonstrated that 126/132 (95\%) of mutations identified were known or predicted tumour drivers and 123/126 (98\%) of these were Tier 1 mutations, which are known to change the activity of the gene product in a way that promotes oncogenic transformation. Results of Cancer Genome Interpreter analysis are shown in Additional file 2: Table S3.

We also investigated the mutational status of two regions of the primary tumor from 50 patients and matched primary and metastatic tumors from 30 patients. Mutations were similar in the different regions from the primary tumor, and also in matched primary and metastatic tumors. Samples taken from two different regions of the same tumor had an overall concordance rate of 94\% (47/50) (Additional file 1: Table S6), indicating a degree of tumor heterogeneity in these samples. Matched primary and metastatic tumors demonstrated an overall concordance rate of 90\% (27/30) (Additional file 1: Table S7). One breast cancer patient had a PTPN11 mutation in the primary tumor that was not identified in the metastatic tumor, another breast cancer patient had an ERBB2 mutation in the metastatic tumor that was not identified in the primary tumor, and one lung cancer patient had a MAP3K13 mutation in the metastatic tumor that was not identified in the primary tumor (Additional file 1: Table S7). The absence of these mutations was verified by droplet digital PCR, an ultrasensitive mutation detection technology. 


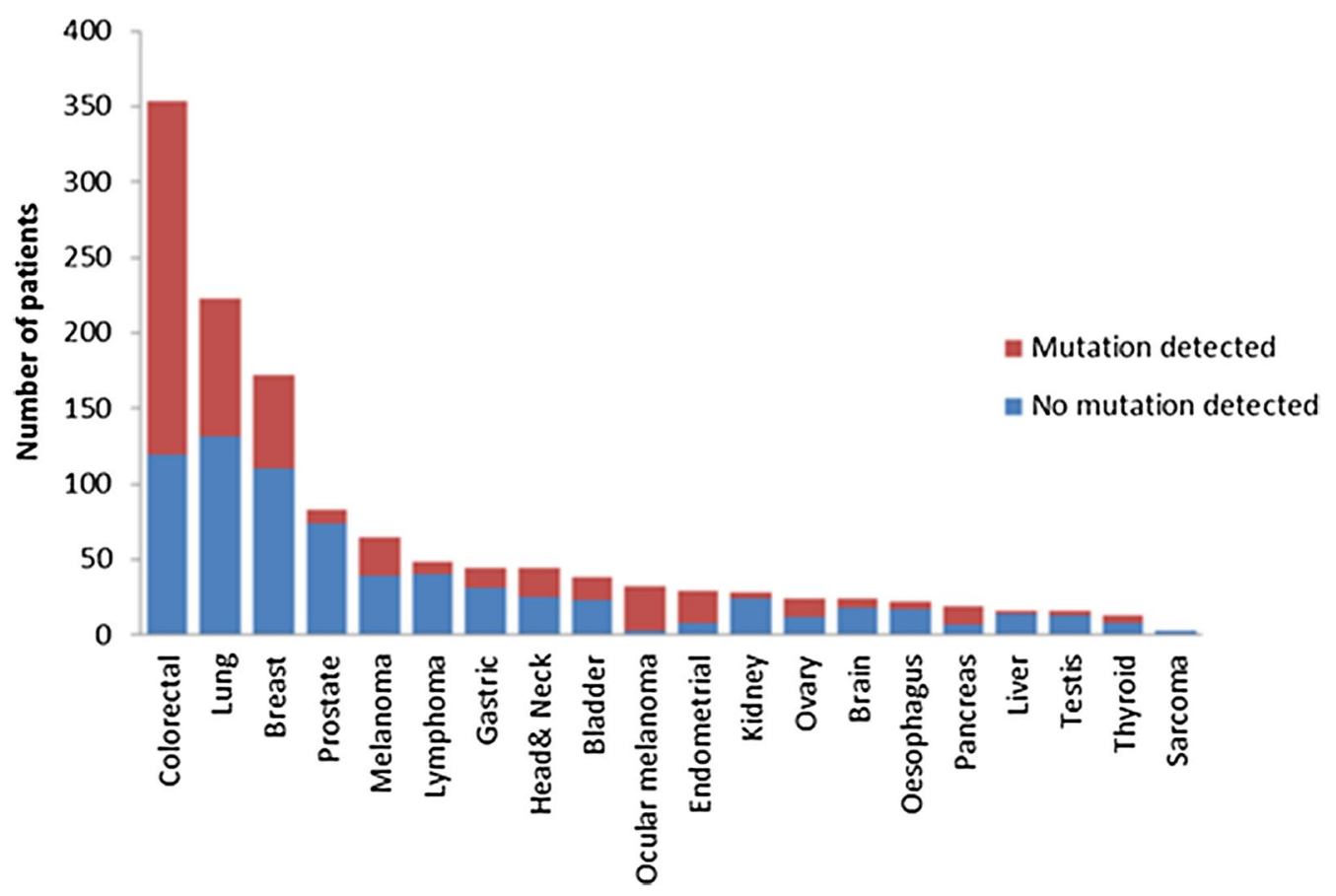

Fig. 1 Number of patients with somatic mutations according to tumor type. The most common tumor type profiled was colorectal, followed by lung, breast, prostate and melanoma. Mutations were most frequently identified in ocular melanoma (90.6\%), endometrial (75.4\%), and colorectal (66.4\%) tumors

\section{Co-occurrence and mutual exclusivity}

Of the 1300 patients analysed, 446 (34.3\%) had a single targetable somatic mutation in their tumors. KRAS was the most common single mutation, and was found in 146 (32.7\%) of these patients. This was followed by PIK3CA mutations, which were present in $114(25.6 \%)$ patients. $101(7.8 \%)$ patients had two targetable mutations in their tumors, while $24(1.8 \%)$ patients had 3 or more targetable mutations in their tumors (Fig. 3a). There was no statistically significant difference in progression free survival (PFS) or overall survival (OS) in patients with no mutations or patients with one or two or more targetable mutations in their tumors (Fig. 3b).

We also examined the co-occurrence and mutual exclusivity of mutations found in our cohort (Fig. 4a). 78.1\% of tumors with mutations (446/571) had only 1 targetable mutation identified, while $21.9 \%$ (125/571) had coexisting mutations. We noted mutual exclusivity between KRAS and BRAF mutations $(\mathrm{p}=0.003)$, and the cooccurrence of somatic mutations in PIK3CA and PTPN11 $(\mathrm{p}=0.019)$, PIK3CA and PTEN $(\mathrm{p}=0.034)$, PTPN11 and $A P C(\mathrm{p}=0.013)$, and PTPN11 and KIT $(\mathrm{p}=0.033)$. We also noted the co-occurrence of mutations in STK11 and $K I T(\mathrm{p}=0.033), F B X W 7$ and $A P C(\mathrm{p}=0.030), F B X W 7$ and HRAS $(\mathrm{p}=0.017), K R A S$ and CTNNB1 $(\mathrm{p}=0.007)$ and HRAS and CTNNB1 ( $\mathrm{p}=0.021)$ (Fig. $4 \mathrm{~b}) . A P C$ and
KRAS mutations co-occurred in 10/152 (6.6\%) of colorectal tumours, with a tendency towards mutual exclusivity. However, it should be noted that only a limited number of $A P C$ mutations were analysed in our study. Somatic mutations in PIK3CA and KRAS frequently cooccurred in colorectal, lung, head and neck, gastric and endometrial tumors (Additional file 1: Table S8).

\section{Correlation of frequent mutations with patient outcomes}

$K R A S, P I K 3 C A$ and BRAF were the three most frequently occurring somatic gene mutations in our cohort. There was no statistically significant difference observed in median PFS or OS between patients with a KRAS mutation in their tumors, and those with no KRAS mutation in their tumors. Similarly the presence of either a PIK3CA or a $B R A F$ mutation had no statistically significant impact on PFS or OS overall (Fig. 5). When mutations were subdivided by pathway, there was no significant difference observed in median PFS in patients with mutations in the MAPK pathway $(\mathrm{p}=0.5956)$ or in patients with mutations in the PI3K pathway $(\mathrm{p}=0.2233)$ compared to patients with tumors wild type for mutations in the corresponding pathway. There was also no difference in overall survival (Fig. 6). However, lung cancer patients whose tumors harboured PI3K pathway mutations had significantly shorter PFS than lung cancer patients 


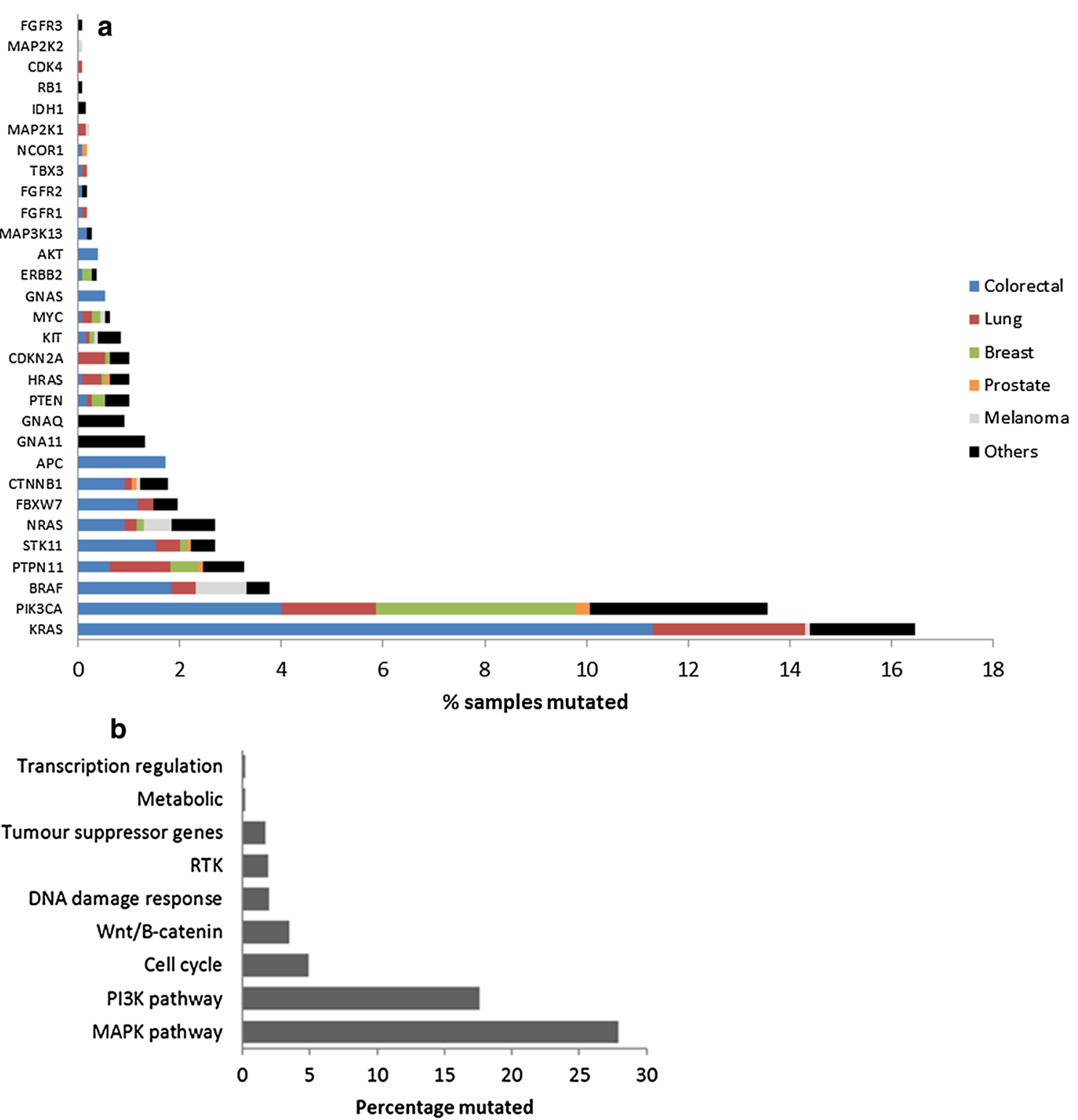

Fig. 2 a Frequency of genomic mutations across human tumor types. Other tumors include lymphoma, gastric, head and neck, bladder, ocular melanoma, endometrial, kidney, ovary, brain, oesophagus, pancreas, liver, testis, thyroid and sarcoma. b Frequency of genomic mutations in human tumor samples by pathway

with PI3K pathway wild type tumors (24.95 months vs. 46 months, $\mathrm{p}=0.047$ ) (Fig. 6).

\section{Effect of aberrations in the PI3K, MAPK and related pathways on PI3K and MAPK pathway activation}

We applied RPPA to determine if genomic mutations in the PI3K, MAPK and related signalling pathway genes activated the PI3K and MAPK pathways. PCA analysis demonstrated no clear difference in pathway activation between mutated and wild type tumors, however proteins were more consistently expressed in the wild type tumors. In contrast, in the mutated tumors, protein expression was more variable, suggesting that there were more proteins activated or repressed in the mutated tumors (Fig. 7).

\section{Discussion}

Next generation sequencing technologies are still not fully cost effective for most clinical laboratories, and hotspot mutational profiling can be accomplished in a more acceptable timeframe for use in routine clinical care. Furthermore, hotspot mutations are more likely to be driver 

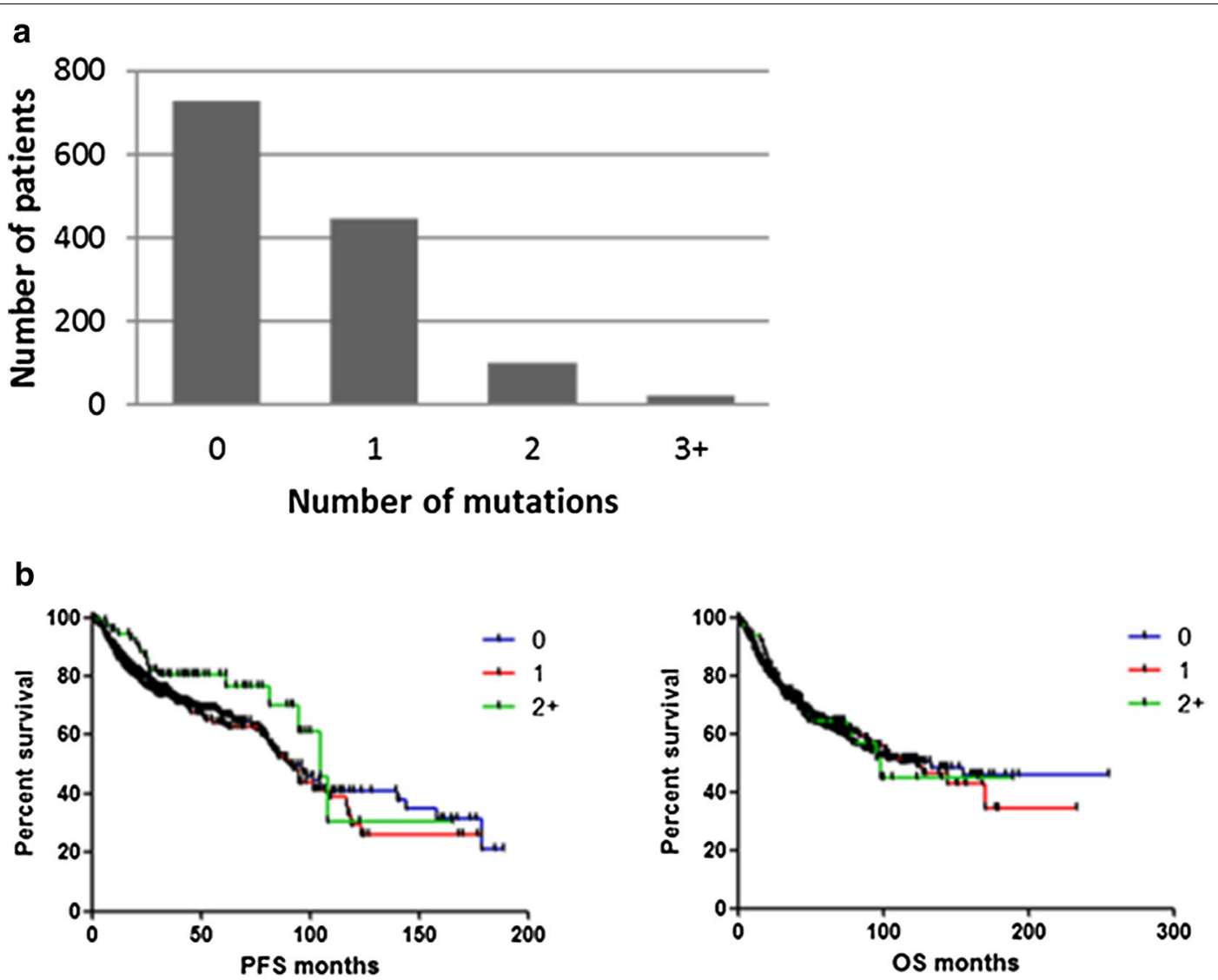

Fig. 3 a Number of genomic mutations per patient tumor. b Correlation between number of mutations and patient survival

mutations and less likely to be incidental genetic variants $[24,25]$. In this study, we have used MassArray analysis to analyse the type and frequency of potentially targetable hotspot somatic mutations in a heterogenous population of solid tumor patients. Activating mutations in the PI3K and MAPK signalling pathways occur frequently in many cancers and have been implicated in the development of resistance to chemotherapy and radiotherapy [26-29]. Therefore, we investigated the frequency of mutations in key gene components of these and related signalling pathways (504 somatic mutations in 47 genes).

Mutations in 30 different genes were identified in $571 / 1300$ (43.9\%) of patients in our study. KRAS and PIK3CA were the most frequently mutated genes, and were identified in $16.5 \%$ and $13.6 \%$ of patients, respectively. There are conflicting reports in the literature about the prognostic value of PIK3CA mutations in solid tumors. While a number of studies have suggested that the presence of a PIK3CA mutation confers a poor prognosis in several cancers including breast, lung and colorectal [30-32], other studies have reported no significant difference between patients harbouring PIK3CA mutations and those with wildtype PIK3CA in their tumors [33-35], which is consistent with our findings. However, there are currently several ongoing trials targeting PIK3CA mutated tumors with PI3K inhibitors, with some promising results. In the Solar-1 trial in hormone receptor positive, HER2 negative advanced breast cancer patients, patients with PIK3CA mutated tumors who received the PI3K inhibitor alpelisib in combination with fulvestrant had significantly improved progression free survival compared to patients who received fulvestrant alone (11 months vs. 5.7 months; $\mathrm{p}=0.00065$ ) [36]. Furthermore, other PI3K inhibitors have also demonstrated anti-tumor responses in patients with advanced solid tumors and non-Hodgkin lymphomas [37, 38].

The frequency of KRAS mutations in our cohort was $16.5 \%$, similar to findings reported in the MSK IMPACT Clinical Sequencing Cohort (16\%) [39]. KRAS has long been considered "undruggable" and for many years has remained an elusive target for drug therapy. However, we have considered KRAS to be potentially targetable, as there are currently several ongoing trials targeting the MAPK pathway in KRAS mutant patients [40]. A 


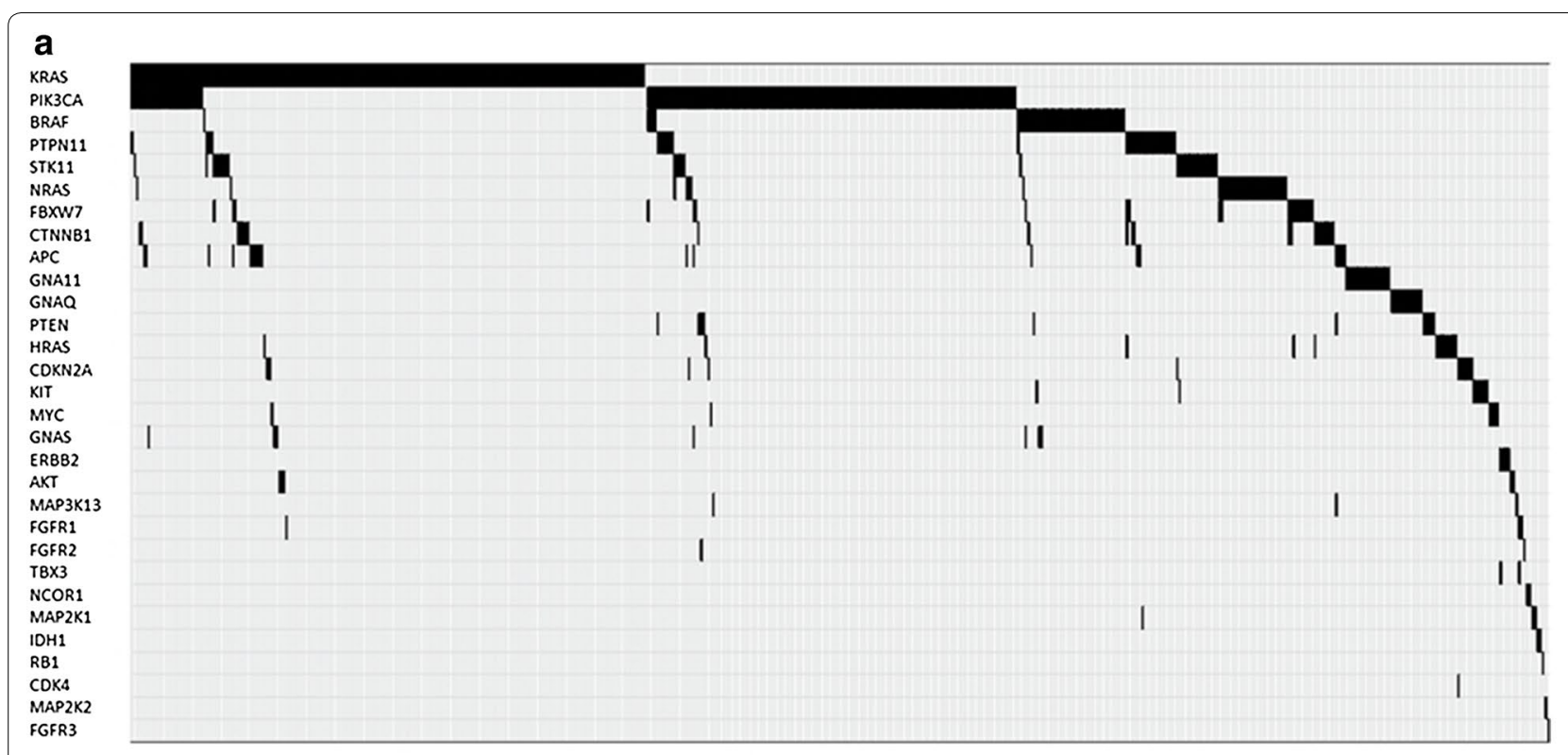

b

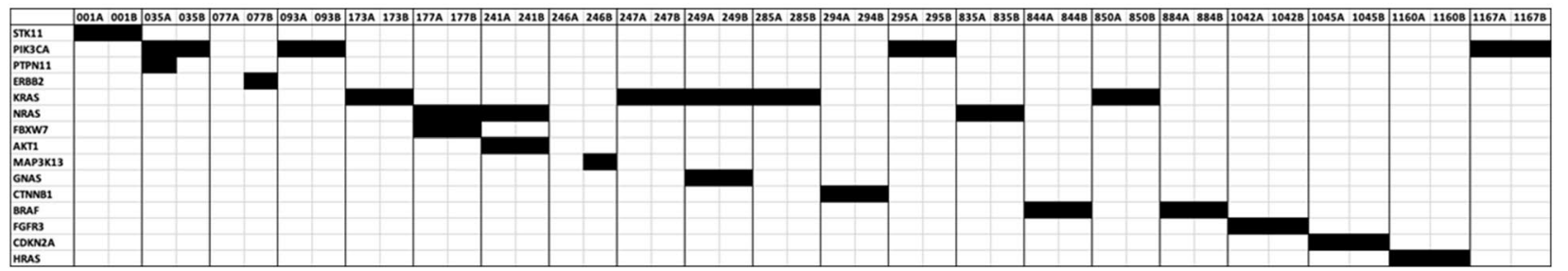

c

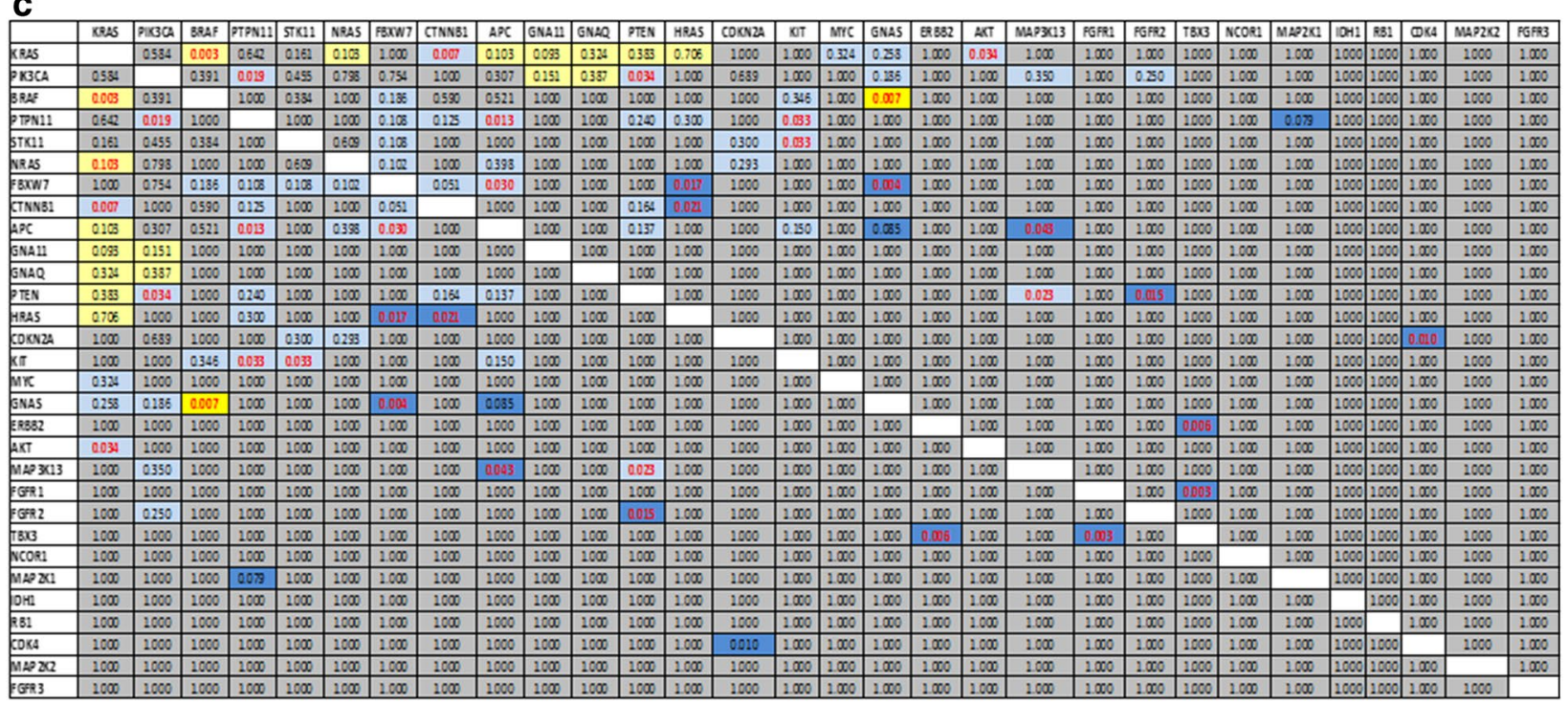

Fig 4 a Mutually exclusive and co-occurring oncogene mutations in human tumors. Mutations were grouped together when the occurred within a given gene. $\mathbf{b}$ Mutation co-occurrence in primary and metastatic pairs. $\mathbf{c}$ Incidence of co-occurring mutations. Grey indicates no association of mutations ( 0.5 odds ratio $<2$ ), Pale yellow indicates some tendency toward mutual exclusivity $(0.1<$ odds ratio $<0.50)$, dark yellow indicates strong tendency toward mutual exclusivity $(0<$ odds ratio $<0.1)$, light blue indicates tendency toward co-occurrence $(2<$ odds ratio $<10)$, dark blue indicates strong tendency toward co-occurrence (odds ratio $>10$ ) 

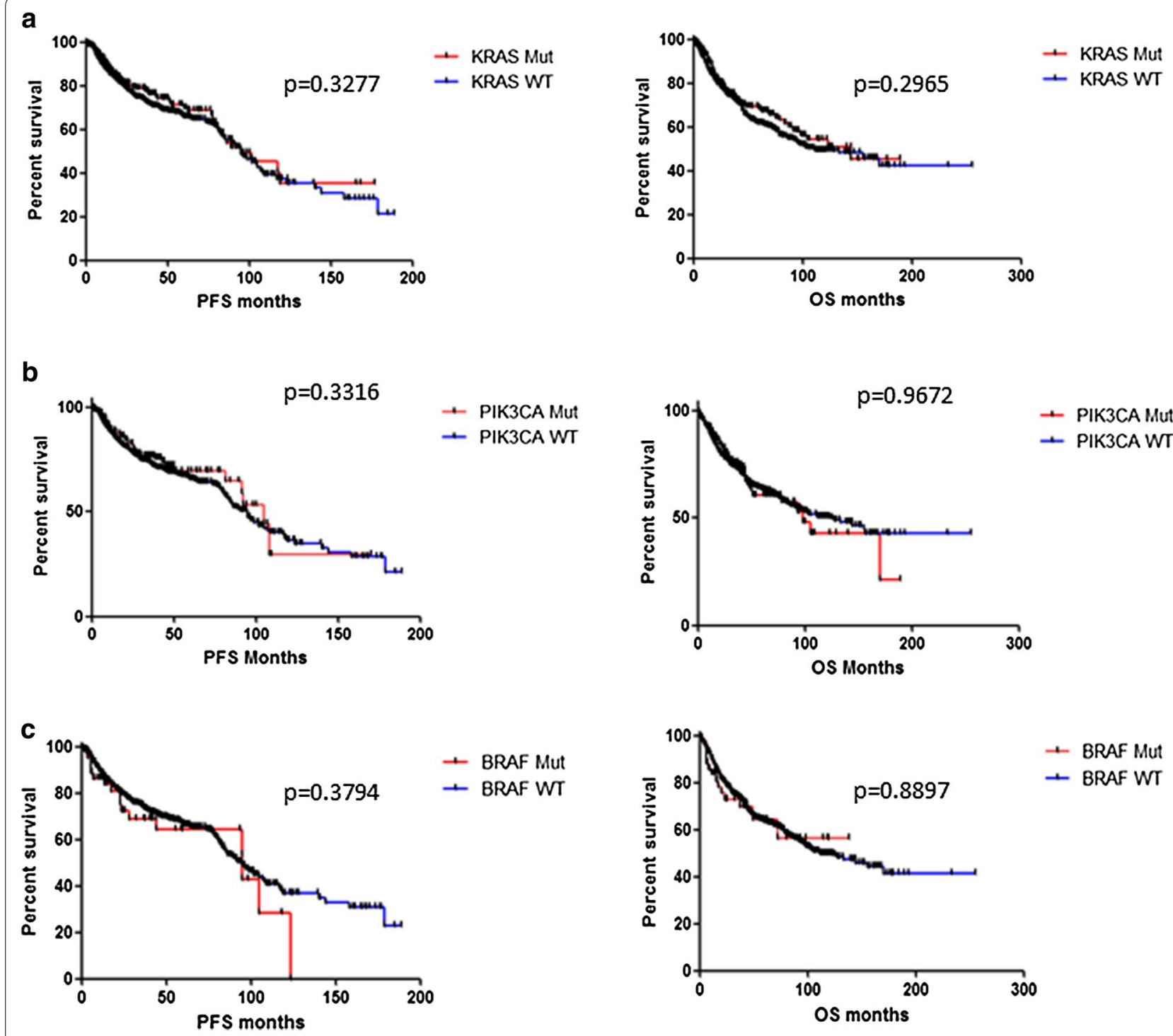

Fig. 5 Correlations between somatic mutation status and patient survival. No significant differences were found in progression free survival (PFS) and overall survival (OS) between patients with a KRAS wild-type tumors and KRAS mutated tumors; $\mathbf{b}$ PIK3CA wild-type and PIK3CA mutated tumors; c BRAF wild-type and BRAF mutated tumors

recent study of AMG-510, a small molecule that specifically and irreversibly inhibits KRAS G12C, demonstrated anti-tumor activity when administered as monotherapy to patients with KRAS G12C mutated advanced solid tumors [41].

In our overall cohort, we did not find any high frequency recurrent mutation to be associated with PFS or OS. Other studies have shown that pathway level alterations rather than recurrent single gene mutations predict response to therapy [42]. Although we did not find alterations in the PI3K or MAPK pathway to be associated with PFS or OS in our overall cohort, we did find that lung cancer patients with somatic PI3K pathway mutations in their tumors had significantly shorter median PFS than patients with PI3K pathway wild type tumors. However, it is important to note that although we found no difference between mutated and non-mutated tumors in terms of OS, $<5 \%$ of our cohort were treated with molecularly targeted therapies.

Colorectal and lung tumors were the two most representative tumor types in our cohort with 354 and 223 patients, respectively. Mutations were identified in $66.4 \%$ of colorectal tumors with KRAS (41.5\%) and PIK3CA (14.7\%) being among the most frequently mutated genes, 

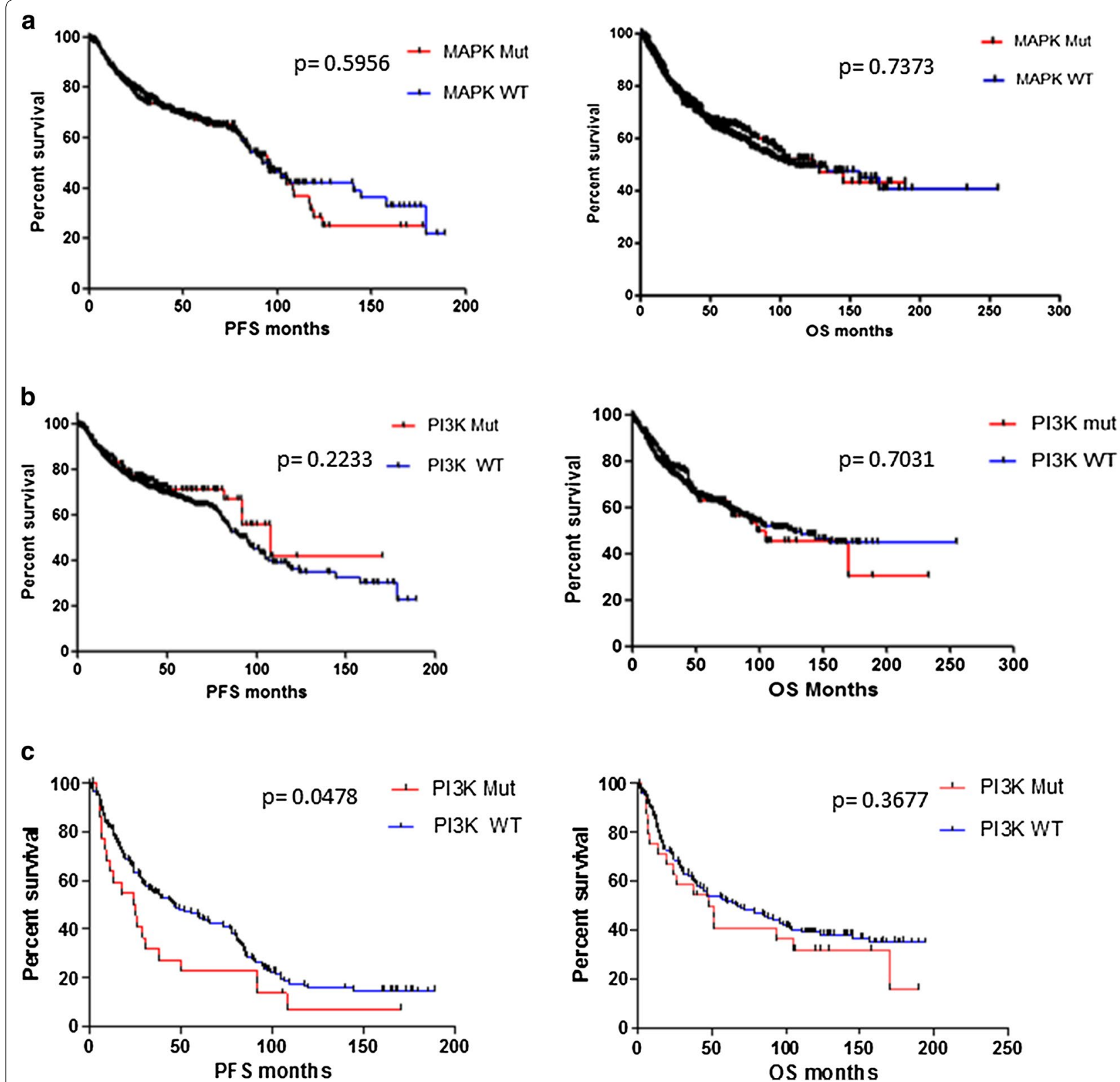

Fig. 6 Correlations between pathway somatic mutation status and patient survival. No significant differences were found in progression free survival (PFS) and overall survival (OS) between patients with a MAPK pathway wild-type tumors and MAPK pathway mutated tumors; $\mathbf{b}$ PI3K pathway wild type and PI3K pathway mutated tumors. c Lung cancer patients with PIK3CA pathway mutated tumors had significantly poorer PFS than lung cancer patients with PI3K pathway wild-type tumors ( 24.95 months vs. 46 months, $p=0.0478$ )

in line with previous studies [43]. The frequency of $A P C$ mutations, which occur at a high frequency in colorectal cancer, in our cohort was lower than previously observed (12.4\%). Although mutations tend to occur within a small part of the APC gene (codons 1286-1513) there are few hotspot mutations [44]. Therefore, our hotspot mutation panel may not be the most appropriate method for identifying $A P C$ mutations. In the lung tumor cohort, mutations were most frequently identified in $K R A S$ (17.5\%), PIK3CA (10.8\%), PTPN11 (5.4\%) and CDKN2A (3.1\%). Almost half $(48.4 \%)$ of our lung cancer patients had lung squamous cell carcinoma (SCC), which is often perceived to lack molecular targets, however our results suggest that KRAS, PIK3CA, PTPN11 and CDKN2A, in particular, could potentially be actionable targets in lung SCC. 

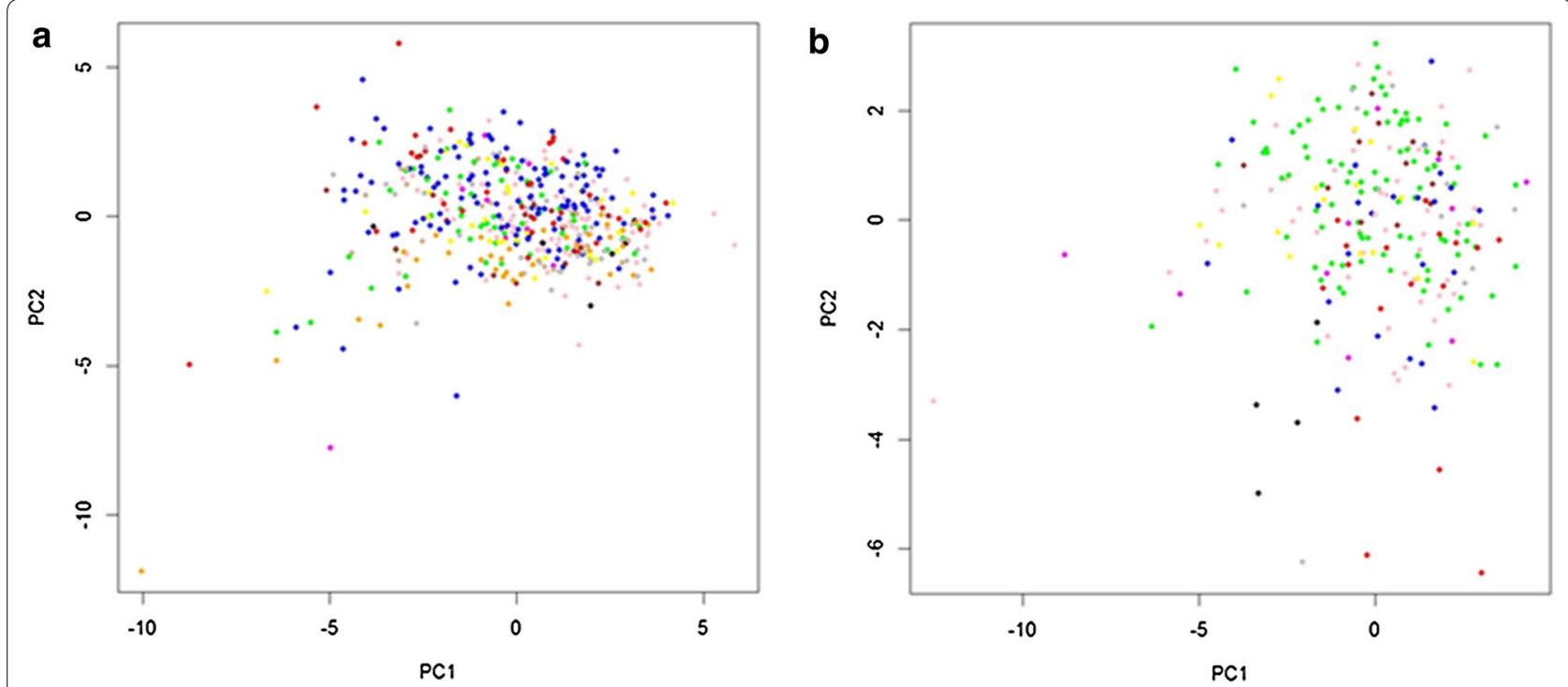

Fig. 7 Principal component analysis (PCA) of RPPA data. a Principal components 1 and 2 for all wild type tumors and $\mathbf{b}$ principal components 1 and 2 for mutated tumors. Blue=bladder, kidney and prostate cancer; Red = breast cancer; Green =colorectal cancer; Grey =cervical, endometrial and ovarian cancer; Black= gastric and oesophageal cancer; Yellow = melanoma and ocular melanoma; Magenta = head and neck cancer; Brown=liver cancer; Orange = lung cancer; $\tan =$ lymphoma; Pink = testicular cancer

One hundred and twenty five (9.6\%) patients in our cohort had co-occurring mutations and interestingly, even using a targeted panel with a limited number of genes, $24(1.8 \%)$ patients had 3 or more genes mutated. Mutation co-occurrence occurred frequently with PIK3CA and KRAS genes in lung, colorectal, head and neck, gastric and endometrial tumors. Previous studies have also shown that mutations in PIK3CA are not mutually exclusive with KRAS mutations and the two are known to commonly co-exist $[45,46]$, confirming the parallel activation of the PI3K and MAPK pathways in the tumors of many patients in our cohort.

Our study also utilised paired samples from two different regions of the same tumor, and from primary and metastatic sites from the same patient, allowing us to determine the concordance of mutations in these samples. We found a high concordance rate between two different regions of the same tumor (94\%) and between the temporally distant recurrent/metastatic site (90\%), suggesting that these mutations are homogenous within the tumor, and agreeing with previous studies that oncogenic drivers are typically shared by all sites of disease, even in patients with heavily pretreated and advanced cancers. Our results suggest that archival tissue as well as newly biopsied samples may be suitable for initial genomic profiling in many patients; however, it is important to note that none of these patients had received targeted therapy. Following targeted therapy, genomic evolution and selection pressure often lead to mediators of acquired resistance becoming the dominant clone as demonstrated by the emergence of the EGFR T790M mutation in EGFR mutated non-small cell lung cancer patients treated with first or second generation tyrosine kinase inhibitors [47]. In this setting liquid biopsies may be more appropriate for molecular stratification of tumors to guide targeted therapy selection.

Currently several ongoing clinical trials are assessing the utility of genomically informed personalised cancer therapy. The NCI-MATCH trial has almost 40 treatment arms investigating targeted therapies against specific genomic aberrations. Some promising results have emerged from these studies so far. The FGFR kinase inhibitor AZD4547 showed some activity, with acceptable toxicities, in various solid tumors harbouring FGFR fusions [48]. Furthermore, the AKT inhibitor capivasertib reduced tumor size in $23 \%$ of patients with $A K T 1$ gene mutations who received the drug. A further $46 \%$ of patients had stable disease [49]. The prospective phase II EXACT trial demonstrated longer PFS when individualised treatment regimens were used and suggested that treatment based on real-time molecular profiling leads to superior clinical benefit [50]. The I-PREDICT study takes into account several actionable molecular alterations in the tumor, with the hypothesis that targeting only one molecular alteration in a tumor is likely to be insufficient to produce a durable anti-tumor response, to propose drug combinations for patients. In this study, $23 \%$ of patients who received matched therapy with nonstandard 
drug combinations, which overall were well tolerated, experienced an objective response [51]. Recently larotrectinib, a pan-TRK inhibitor, and entrectinib, a panTRK, ALK, and ROS1 tyrosine kinase inhibitor, were the first tumor-agnostic-targeted therapies developed and approved in oncology for the treatment of pediatric and adult tumors that have an NTRK gene fusion [52].

Functional proteomic analysis using RPPA showed that although the PI3K and MAPK signalling pathways are more altered in mutated tumors than in wild type, all of the tumors have alterations in these signalling pathways regardless of the mutation status of the tumor or the type of mutation. This suggests that even in the absence of a specific genomic mutation, these patients could potentially benefit from treatment with a multitargeted tyrosine kinase inhibitor (TKI). There are several multitargeted drugs already used in the treatment of various cancer types [53]. One such drug, regorafenib, improves PFS and OS in patients with refractory colorectal cancer independent of tumor mutation status [54].

We are aware that our study has several limitations. Only hotspot mutations in specific genes of interest were evaluated, making the method unsuitable for genes like $A P C$, which are highly mutated but have few hotspots. Secondly, because the majority of our samples were primary tumors only a minority of patients received targeted therapies in this setting, making it impossible to determine the impact of targetable somatic mutations on response to targeted therapy. The number of paired primary/metastatic samples in our study was limited, and although we observed high concordance between the primary tumor and recurrence/ metastatic site, these were all first recurrences and concordance is likely to be less at later recurrence, particularly in patients who have received targeted therapies. Furthermore, our study does not examine epigenetic and transcriptomic changes involved in cancer pathogenesis. Disruption of key epigenetic regulators by mutation leads to an altered transcriptome, which can multiply the effect of a single genetic alteration [55]. Therefore, complete understanding of transcriptomic and epigenomic changes in the tumor could compliment the molecular information obtained from hotspot mutation profiling, as described herein.

\section{Conclusions}

Although the clinical utility of genomic profiling of tumors has not been fully demonstrated, cancer treatment is moving to a new paradigm where the molecular characteristics of the tumor are used to inform treatment decisions. Larotrectinib, entrectinib and pembrolizumab have been approved across cancer types based solely on the mutational status of the tumor $[56,57]$. The present study aimed to identify key alterations that may represent important targets for novel therapies. Although not without its limitations, using a hotspot mutation profiling approach avoids complex NGS designs and bioinformatics analysis, can be accomplished in a realistic timeframe for use in day-to-day clinical care, and could be used to expand treatment options for patients with cancer.

\section{Supplementary information}

Supplementary information accompanies this paper at https://doi. org/10.1186/s12967-020-02273-4.

Additional file 1: Figure S1. Breakdown of tumour types in A. Colorectal B. Lung and C. Breast tumour cohorts. Table S1. List of mutations analysed using the Agena MassArray technology. Table S2. Somatic mutations tested subdivided by pathway. Table S4. Antibodies used for Reverse Phase Protein Array (RPPA), including the company from which it was purchased, the catalog number, the host species and the dilution at which it was used. Table S6. Somatic mutation status in samples taken from two different regions of the same primary tumour. Samples identified in one sample but not in the other are identified in bold print. Table S7. Somatic mutation status of primary and matched metastatic tumour samples. Samples identified in one sample but not in the other are identified in bold print. Table S8. Frequency and co-occurrence of somatic PIK3CA and KRAS mutations in solid tumour samples.

Additional file 2: Table S3. Cancer Genome Interpreter mutation analysis of the mutations identified in solid tumour samples analysed in our study.

Additional file 3: Table S5. Percentage of somatic hotspot mutations in each tumour type.

\section{Abbreviations}

DNA: Deoxyribonucleic Acid; FFPE: Formalin Fixed Paraffin Embedded; FISH: Fluorescence in situ hybridisation; MAPK: Mitogen-activated protein kinase; NGS: Next generation sequencing; OS: Overall survival; PCA: Principal component analysis; PFS: Progression free survival; PI3K: Phosphoinositide 3-kinase; RPPA: Reverse Phase Protein Array; TKI: Tyrosine kinase inhibitor.

\section{Acknowledgements}

Non-applicable.

\section{Authors' contributions}

$\mathrm{BH}$ conceived the study and co-ordinated the experiments and revised the manuscript. JC conceived the study and revised the manuscript. ST COordinated the experiments, performed the experiments, analysed the data and wrote the manuscript. AC, MJZ, YE, SR, KIAJ, KG, AE, JF, RC, AOG, and RK carried out genomic analysis. MC, CM and AF carried out the RPPA analysis. SM performed statistical analysis, EWK, SK, KOB, LG, OB, PM, NOD, FK, AOR and MD performed analysis of the data generated. All authors read and approved the final manuscript.

\section{Funding}

This work was supported by Science Foundation Ireland-funded Molecular Therapeutics for Cancer Ireland (08-SRC-B1410), the Health Research Board (HRA/POR2012/054), the North East Cancer Research and Education Trust (NECRET), and the Fox and Kerins Families.

\section{Availability of data and materials}

All data generated or analyzed during this study are included in this published article. Raw and processed data are stored in the laboratory of $\mathrm{BH}$ and are available upon request. 


\section{Ethics approval and consent to participate}

Formalin-fixed paraffin embedded tumor samples were obtained from tumor banks under the auspices of Institutional Review Board-approved protocols at Beaumont Hospital Dublin, Ireland, St. Vincent's University Hospital, incorporating the Royal Victoria Eye and Ear Hospital, Dublin, Ireland and St. James's Hospital, Dublin, Ireland.

\section{Consent for publication}

Non-applicable.

\section{Competing interests}

The authors declare that they have no competing interests.

\begin{abstract}
Author details
${ }^{1}$ Medical Oncology Lab, Department of Molecular Medicine, Royal College of Surgeons in Ireland, RCSI Smurfit Building, Beaumont Hospital, Dublin, Ireland. ${ }^{2}$ Department of Medical Oncology, St. James's Hospital Dublin, Dublin, Ireland. ${ }^{3}$ Data Science Centre, Royal College of Surgeons in Ireland, Dublin, Ireland. ${ }^{4}$ Department of Pathology, Royal College of Surgeons in Ireland, Dublin, Ireland. ${ }^{5}$ Department of Pathology, St. Vincent's University Hospital, Dublin, Ireland. ${ }^{6}$ Department of Pathology, Royal Victoria Eye and Ear Hospital, Dublin, Ireland. ${ }^{7}$ Princess Alexandra Hospital, Brisbane, Australia. ${ }^{8}$ Department of Medical Oncology, Beaumont Hospital, Dublin, Ireland. ${ }^{9}$ National Institute for Cellular Biotechnology, Dublin City University, Dublin, Ireland. ${ }^{10}$ Department of Medical Oncology, St. Vincent's University Hospital, Dublin, Ireland.
\end{abstract}

Received: 6 September 2019 Accepted: 14 February 2020

Published online: 22 February 2020

\section{References}

1. Hirota S, Isozaki K, Moriyama Y, Hashimoto K, Nishida T, Ishiguro S, et al. Gain-of-function mutations of c-kit in human gastrointestinal stromal tumors. Science. 1998;279(5350):577-80.

2. Pao W, Miller V, Zakowski M, Doherty J, Politi K, Sarkaria I, et al. EGF receptor gene mutations are common in lung cancers from "never smokers" and are associated with sensitivity of tumors to gefitinib and erlotinib. Proc Natl Acad Sci USA. 2004;101(36):13306-11.

3. Khambata-Ford S, Garrett CR, Meropol NJ, Basik M, Harbison CT, Wu S, et al. Expression of epiregulin and amphiregulin and K-ras mutation status predict disease control in metastatic colorectal cancer patients treated with cetuximab. J Clin Oncol. 2007;25(22):3230-7.

4. de Jong FA, Verweij J. Role of imatinib mesylate (Gleevec/Glivec) in gastrointestinal stromal tumors. Expert Rev Anticancer Ther. 2003;3(6):757-66

5. Paez JG, Janne PA, Lee JC, Tracy S, Greulich H, Gabriel S, et al. EGFR mutations in lung cancer: correlation with clinical response to gefitinib therapy. Science. 2004;304(5676):1497-500.

6. Chapman PB, Hauschild A, Robert C, Haanen JB, Ascierto P, Larkin J, et al. Improved survival with vemurafenib in melanoma with BRAF V600E mutation. N Engl J Med. 2011;364(26):2507-16.

7. Ascierto PA, Minor D, Ribas A, Lebbe C, O'Hagan A, Arya N, et al. Phase II trial (BREAK-2) of the BRAF inhibitor dabrafenib (GSK2118436) in patients with metastatic melanoma. J Clin Oncol. 2013;31(26):3205-11.

8. Hauschild A, Grob JJ, Demidov LV, Jouary T, Gutzmer R, Millward M, et al. Dabrafenib in BRAF-mutated metastatic melanoma: a multicentre, open-label, phase 3 randomised controlled trial. Lancet. 2012;380(9839):358-65.

9. Cunningham D, Humblet $Y$, Siena S, Khayat D, Bleiberg H, Santoro A, et al. Cetuximab monotherapy and cetuximab plus irinotecan in irinotecanrefractory metastatic colorectal cancer. N Engl J Med. 2004;351(4):337-45.

10. Douillard JY, Siena S, Cassidy J, Tabernero J, Burkes R, Barugel M, et al. Randomized, phase III trial of panitumumab with infusional fluorouracil, leucovorin, and oxaliplatin (FOLFOX4) versus FOLFOX4 alone as first-line treatment in patients with previously untreated metastatic colorectal cancer: the PRIME study. J Clin Oncol. 2010;28(31):4697-705.

11. Carlisle JW, Ramalingam SS. Role of osimertinib in the treatment of EGFR-mutation positive non-small-cell lung cancer. Future Oncol. 2019:15(8):805-16.
12. Ricciuti B, Baglivo S, De Giglio A, Chiari R. Afatinib in the first-line treatment of patients with non-small cell lung cancer: clinical evidence and experience. Ther Adv Respir Dis. 2018;12:1753466618808659.

13. Trojaniello C, Festino L, Vanella V, Ascierto PA. Encorafenib in combination with binimetinib for unresectable or metastatic melanoma with BRAF mutations. Expert Rev Clin Pharmacol. 2019;12(3):259-66.

14. Greenman C, Stephens P, Smith R, Dalgliesh GL, Hunter C, Bignell G, et al. Patterns of somatic mutation in human cancer genomes. Nature. 2007:446(7132):153-8.

15. MacConaill LE, Campbell CD, Kehoe SM, Bass AJ, Hatton C, Niu L, et al. Profiling critical cancer gene mutations in clinical tumor samples. PLoS ONE. 2009;4(11):e7887.

16. Thomas RK, Baker AC, Debiasi RM, Winckler W, Laframboise T, Lin WM, et al. High-throughput oncogene mutation profiling in human cancer. Nat Genet. 2007;39(3):347-51.

17. Fleitas T, Ibarrola-Villava M, Ribas G, Cervantes A. MassARRAY determination of somatic oncogenic mutations in solid tumors: moving forward to personalized medicine. Cancer Treat Rev. 2016;49:57-64.

18. Ibarrola-Villava M, Fleitas T, Llorca-Cardenosa MJ, Mongort C, Alonso E, Navarro S, et al. Determination of somatic oncogenic mutations linked to target-based therapies using MassARRAY technology. Oncotarget. 2016;7(16):22543-55

19. Dienstmann R, Serpico D, Rodon J, Saura C, Macarulla T, Elez E, et al. Molecular profiling of patients with colorectal cancer and matched targeted therapy in phase I clinical trials. Mol Cancer Ther. 2012;11(9):2062-71.

20. Beadling C, Heinrich MC, Warrick A, Forbes EM, Nelson D, Justusson E, et al. Multiplex mutation screening by mass spectrometry evaluation of 820 cases from a personalized cancer medicine registry. J Mol diagn. 2011;13(5):504-13.

21. Guo H, Liu W, Ju Z, Tamboli P, Jonasch E, Mills GB, et al. An efficient procedure for protein extraction from formalin-fixed, paraffin-embedded tissues for reverse phase protein arrays. Proteome Sci. 2012;10(1):56.

22. Elster N, Cremona M, Morgan C, Toomey S, Carr A, O'Grady A, et al. A preclinical evaluation of the PI3K alpha/delta dominant inhibitor BAY 80-6946 in HER2-positive breast cancer models with acquired resistance to the HER2-targeted therapies trastuzumab and lapatinib. Breast Cancer Res Treat. 2015;149(2):373-83.

23. Hennessy BT, Lu Y, Gonzalez-Angulo AM, Carey MS, Myhre S, Ju Z, et al. A technical assessment of the utility of reverse phase protein arrays for the study of the functional proteome in non-microdissected human breast cancers. Clin Proteom. 2010:6(4):129-51.

24. Chen T, Wang Z, Zhou W, Chong Z, Meric-Bernstam F, Mills GB, et al. Hotspot mutations delineating diverse mutational signatures and biological utilities across cancer types. BMC Genomics. 2016;17(Suppl 2):394.

25. Bailey MH, Tokheim C, Porta-Pardo E, Sengupta S, Bertrand D, Weerasinghe $A$, et al. Comprehensive characterization of cancer driver genes and mutations. Cell. 2018;173(2):371-385.e18.

26. Hennessy BT, Smith DL, Ram PT, Lu Y, Mills GB. Exploiting the PI3K/ AKT pathway for cancer drug discovery. Nat Rev Drug Discov. 2005:4(12):988-1004.

27. De Luca A, Maiello MR, D'Alessio A, Pergameno M, Normanno N. The RAS/RAF/MEK/ERK and the PI3K/AKT signalling pathways: role in cancer pathogenesis and implications for therapeutic approaches. Expert Opin Ther Targets. 2012;16(Suppl 2):S17-27.

28. Ihle NT, Byers LA, Kim ES, Saintigny P, Lee JJ, Blumenschein GR, et al. Effect of KRAS oncogene substitutions on protein behavior: implications for signaling and clinical outcome. J Natl Cancer Inst. 2012;104(3):228-39.

29. Abdul-Jalil KI, Sheehan KM, Toomey S, Schmid J, Prehn J, O'Grady A, et al. The frequencies and clinical implications of mutations in 33 kinaserelated genes in locally advanced rectal cancer: a pilot study. Ann Surg Oncol. 2014;21(8):2642-9.

30. Loibl S, Majewski I, Guarneri V, Nekljudova V, Holmes E, Bria E, et al. PIK3CA mutations are associated with reduced pathological complete response rates in primary HER2-positive breast cancer: pooled analysis of 967 patients from five prospective trials investigating lapatinib and trastuzumab. Ann Oncol. 2016;27(8):1519-25.

31. Song $Z, Y u X$, Zhang $Y$. Mutation and prognostic analyses of PIK3CA in patients with completely resected lung adenocarcinoma. Cancer Med. 2016;5(10):2694-700 
32. Ogino S, Nosho K, Kirkner GJ, Shima K, Irahara N, Kure S, et al. PIK3CA mutation is associated with poor prognosis among patients with curatively resected colon cancer. J Clin Oncol. 2009;27(9):1477-84.

33. Mei ZB, Duan CY, Li CB, Cui L, Ogino S. Prognostic role of tumor PIK3CA mutation in colorectal cancer: a systematic review and meta-analysis. Ann Oncol. 2016;27(10):1836-48.

34. Ibrahim EM, Kazkaz GA, Al-Mansour MM, Al-Foheidi ME. The predictive and prognostic role of phosphatase phosphoinositol-3 (PI3) kinase (PIK3CA) mutation in HER2-positive breast cancer receiving HER2-targeted therapy: a meta-analysis. Breast Cancer Res Treat. 2015;152(3):463-76.

35. Scheffler M, Bos M, Gardizi M, Konig K, Michels S, Fassunke J, et al. PIK3CA mutations in non-small cell lung cancer (NSCLC): genetic heterogeneity, prognostic impact and incidence of prior malignancies. Oncotarget. 2015;6(2):1315-26.

36. Andre F, Ciruelos E, Rubovszky G, Campone M, Loibl S, Rugo HS, et al. Alpelisib for PIK3CA-mutated, hormone receptor-positive advanced breast cancer. N Engl J Med. 2019;380(20):1929-40.

37. Patnaik A, Appleman LJ, Tolcher AW, Papadopoulos KP, Beeram M, Rasco DW, et al. First-in-human phase I study of copanlisib (BAY 80-6946), an intravenous pan-class I phosphatidylinositol 3-kinase inhibitor, in patients with advanced solid tumors and non-Hodgkin's lymphomas. Ann Oncol. 2016;27(10):1928-40

38. Juric D, Rodon J, Tabernero J, Janku F, Burris HA, Schellens JHM, et al. Phosphatidylinositol 3-kinase alpha-selective inhibition with alpelisib (BYL719) in PIK3CA-altered solid tumors: results from the first-in-human study. J Clin Oncol. 2018;36(13):1291-9.

39. Zehir A, Benayed R, Shah RH, Syed A, Middha S, Kim HR, et al. Mutational landscape of metastatic cancer revealed from prospective clinical sequencing of 10,000 patients. Nat Med. 2017;23(6):703-13.

40. Hymowitz SG, Malek S. Targeting the MAPK pathway in RAS mutant cancers. Cold Spring Harb Perspect Med. 2018. https://doi.org/10.1101/ cshperspect.a031492.

41. Fakih M, O’Neil B, Price TJ, Falchook GS, Desai J, Kuo J, et al. Phase 1 study evaluating the safety, tolerability, pharmacokinetics (PK), and efficacy of AMG 510, a novel small molecule KRASG12C inhibitor, in advanced solid tumors. J Clin Oncol. 2019;37(15_suppl):3003.

42. Shi W, Jiang T, Nuciforo P, Hatzis $C$, Holmes $E$, Harbeck N, et al. Pathway level alterations rather than mutations in single genes predict response to HER2-targeted therapies in the neo-ALTTO trial. Ann Oncol. 2017;28(1):128-35.

43. Cancer Genome Atlas Network. Comprehensive molecular characterization of human colon and rectal cancer. Nature. 2012;487(7407):330-7.

44. Miyoshi $Y$, Nagase $H$, Ando $H$, Horii A, Ichii S, Nakatsuru S, et al. Somatic mutations of the APC gene in colorectal tumors: mutation cluster region in the APC gene. Hum Mol Genet. 1992;1(4):229-33.

45. Janku F, Lee JJ, Tsimberidou AM, Hong DS, Naing A, Falchook GS, et al. PIK3CA mutations frequently coexist with RAS and BRAF mutations in patients with advanced cancers. PLoS ONE. 2011;6(7):e22769.
46. Wang $L, H u H$ Pan $Y$ Wang $R$, LiY Shen $L$ et al. PIK3CA mutations frequently coexist with EGFR/KRAS mutations in non-small cell lung cancer and suggest poor prognosis in EGFR/KRAS wildtype subgroup. PLOS ONE. 2014;9(2):e88291

47. Hata AN, Niederst MJ, Archibald HL, Gomez-Caraballo M, Siddiqui FM, Mulvey HE, et al. Tumor cells can follow distinct evolutionary paths to become resistant to epidermal growth factor receptor inhibition. Nat Med. 2016;22(3):262-9.

48. Chae YK, Vaklavas C, Cheng HH, Hong F, Harris L, Mitchell EP, Zwiebel JA, McShane L, Gray RJ, Li S, Ivy SP, Ansher SS, Hamilton SR, Williams PM, Tricoli JV, Arteaga CL, Conley BA, O'Dwyer PJ, Chen AP, Flaherty K. Molecular analysis for therapy choice (MATCH) arm W: phase II study of AZD4547 in patients with tumors with aberrations in the FGFR pathway. J Clin Oncol. 2018. https://doi.org/10.1200/JCO.2018.36.15 suppl.2503.

49. Hyman DM, Smyth LM, Donoghue MTA, Westin SN, Bedard PL, et al. AKT inhibition in solid tumours with AKT1 mutations. J Clin Oncol. 2017;35(20):2251-9.

50. Prager GW, Unseld M, Waneck F, Mader R, Wrba F, Raderer M, et al. Results of the extended analysis for cancer treatment (EXACT) trial: a prospective translational study evaluating individualized treatment regimens in oncology. Oncotarget. 2019;10(9):942-52.

51. Sicklick JK, Kato S, Okamura R, Schwaederle M, Hahn ME, Williams CB, et al. Molecular profiling of cancer patients enables personalized combination therapy: the I-PREDICT study. Nat Med. 2019;25(5):744-50.

52. Saleh $K$, Khalifeh-Saleh N, Kourie HR. TRK inhibitors: toward an era of agnostic targeted therapies in oncology. Pharmacogenomics. 2019;20(13):927-9.

53. Ferguson FM, Gray NS. Kinase inhibitors: the road ahead. Nat Rev Drug Discov. 2018;17(5):353-77.

54. Grothey A, Van Cutsem E, Sobrero A, Siena S, Falcone A, Ychou M, et al. Regorafenib monotherapy for previously treated metastatic colorectal cancer (CORRECT): an international, multicentre, randomised, placebocontrolled, phase 3 trial. Lancet. 2013;381(9863):303-12.

55. Shen H, Laird PW. Interplay between the cancer genome and epigenome. Cell. 2013;153(1):38-55.

56. Drilon A, Laetsch TW, Kummar S, DuBois SG, Lassen UN, Demetri GD, et al. Efficacy of larotrectinib in TRK fusion-positive cancers in adults and children. N Engl J Med. 2018;378(8):731-9.

57. Le DT, Uram JN, Wang $H$, Bartlett BR, Kemberling $H$, Eyring AD, et al. PD-1 blockade in tumors with mismatch-repair deficiency. N Engl J Med. 2015;372(26):2509-20.

\section{Publisher's Note}

Springer Nature remains neutral with regard to jurisdictional claims in published maps and institutional affiliations.
Ready to submit your research? Choose BMC and benefit from:

- fast, convenient online submission

- thorough peer review by experienced researchers in your field

- rapid publication on acceptance

- support for research data, including large and complex data types

- gold Open Access which fosters wider collaboration and increased citations

- maximum visibility for your research: over $100 \mathrm{M}$ website views per year

At BMC, research is always in progress.

Learn more biomedcentral.com/submissions 